\title{
Does intrathecal nicardipine for cerebral vasospasm following subarachnoid hemorrhage correlate with reduced delayed cerebral ischemia? A retrospective propensity score-based analysis
}

\author{
Ofer Sadan, MD, PhD, ${ }^{1}$ Hannah Waddel, BS, ${ }^{2}$ Reneé Moore, PhD, ${ }^{2}$ Chen Feng, PhD, ${ }^{3}$ \\ Yajun Mei, PhD, ${ }^{3}$ David Pearce, MD, Jacqueline Kraft, MD, ${ }^{1}$ Cederic Pimentel, MD, ${ }^{1}$ \\ Subin Mathew, MD, ${ }^{1}$ Feras Akbik, MD, PhD, ${ }^{1}$ Pouya Ameli, MD, ${ }^{1}$ Alexis Taylor, MD, ${ }^{1}$ \\ Lisa Danyluk, MSN, ${ }^{4}$ Kathleen S. Martin, DNP, ${ }^{4}$ Krista Garner, DNP, ${ }^{4}$ Jennifer Kolenda, DNP, \\ Amit Pujari, MD, ${ }^{5}$ William Asbury, PharmD, ${ }^{6}$ Blessing N. R. Jaja, MD, PhD, ${ }^{7}$ \\ R. Loch Macdonald, MD, PhD, ${ }^{8}$ C. Michael Cawley, MD, ${ }^{9}$ Daniel L. Barrow, MD, ${ }^{9}$ and \\ Owen Samuels, MD'
}

1Department of Neurology and Neurosurgery, Division of Neurocritical Care, Emory University School of Medicine, Atlanta, Georgia; '2Department of Biostatistics and Bioinformatics, Biostatistics Collaboration Core, Emory University, Atlanta, Georgia; ${ }^{3} \mathrm{H}$. Milton Stewart School of Industrial and Systems Engineering, Georgia Institute of Technology, Atlanta, Georgia; ${ }^{4}$ Neuroscience ICU, and ${ }^{6}$ Department of Clinical Pharmacy, Emory Healthcare, Atlanta, Georgia; ${ }^{5}$ Emory University School of Medicine, Atlanta, Georgia; ' 7 Department of Genetics and Development, Toronto Western Hospital, University Health Network, Toronto, Ontario, Canada; ${ }^{8}$ Department of Neurological Surgery, UCSF Fresno, California; and ${ }^{9}$ Department of Neurosurgery, Emory University Hospital and School of Medicine, Atlanta, Georgia

OBJECTIVE Cerebral vasospasm and delayed cerebral ischemia $(\mathrm{DCl})$ contribute to poor outcome following subarachnoid hemorrhage (SAH). With the paucity of effective treatments, the authors describe their experience with intrathecal (IT) nicardipine for this indication.

METHODS Patients admitted to the Emory University Hospital neuroscience ICU between 2012 and 2017 with nontraumatic SAH, either aneurysmal or idiopathic, were included in the analysis. Using a propensity-score model, this patient cohort was compared to patients in the Subarachnoid Hemorrhage International Trialists (SAHIT) repository who did not receive IT nicardipine. The primary outcome was $\mathrm{DCl}$. Secondary outcomes were long-term functional outcome and adverse events.

RESULTS The analysis included 1351 patients, 422 of whom were diagnosed with cerebral vasospasm and treated with IT nicardipine. When compared with patients with no vasospasm $(n=859)$, the treated group was significantly younger (mean age $51.1 \pm 12.4$ years vs $56.7 \pm 14.1$ years, $p<0.001$ ), had a higher World Federation of Neurosurgical Societies score and modified Fisher grade, and were more likely to undergo clipping of the ruptured aneurysm as compared to endovascular treatment $(30.3 \%$ vs $11.3 \%, p<0.001)$. Treatment with IT nicardipine decreased the daily mean transcranial Doppler velocities in $77.3 \%$ of the treated patients. When compared to patients not receiving IT nicardipine, treatment was not associated with an increased rate of bacterial ventriculitis $(3.1 \%$ vs $2.7 \%, p>0.1)$, yet higher rates of ventriculoperitoneal shunting were noted $(19.9 \%$ vs $8.8 \%, p<0.01)$. In a propensity score comparison to the SAHIT database, the odds ratio (OR) to develop DCI with IT nicardipine treatment was 0.61 (95\% confidence interval [Cl] 0.44-0.84), and the OR to have a favorable functional outcome (modified Rankin Scale score $\leq 2)$ was 2.17 (95\% Cl 1.61-2.91).

\footnotetext{
ABBREVIATIONS $\mathrm{ACA}=$ anterior cerebral artery; $\mathrm{BA}=$ basilar artery; $\mathrm{Cl}=$ confidence interval; $\mathrm{CTA}=\mathrm{CT}$ angiography; $\mathrm{DCl}=$ delayed cerebral ischemia; $\mathrm{DSA}=$ digital subtraction angiography; EVD = external ventricular drain; IQR = interquartile range; ISAT = International Subarachnoid Aneurysm Trial; IT = intrathecal; $M C A=$ middle cerebral artery; mRS = modified Rankin Scale; NEWTON = Nimodipine Microparticles to Enhance Recovery While Reducing Toxicity After Subarachnoid Hemorrhage; OR = odds ratio; $\mathrm{SAH}=$ subarachnoid hemorrhage; $\mathrm{SAHIT}=$ Subarachnoid Hemorrhage International Trialists; $\mathrm{TCD}$ = transcranial Doppler; VPS = ventriculoperitoneal shunt; WFNS $=$ World Federation of Neurosurgical Societies
}

SUBMITTED October 5, 2020. ACCEPTED December 14, 2020

INCLUDE WHEN CITING Published online June 4, 2021; DOI: 10.3171/2020.12.JNS203673. 
CONCLUSIONS IT nicardipine was associated with improved outcome and reduced $\mathrm{DCl}$ compared with propensitymatched controls. There was an increased need for permanent CSF diversion but no other safety issues. These data should be considered when selecting medications and treatments to study in future randomized controlled clinical trials for SAH.

https://thejns.org/doi/abs/10.3171/2020.12.JNS203673

KEYWORDS subarachnoid hemorrhage; delayed cerebral ischemia; vasospasm; calcium channel blocker; intrathecal administration; vascular disorders

$\mathrm{N}$ ONTRAUMATIC subarachnoid hemorrhage (SAH) is an uncommon, but often devastating, cause of stroke that disproportionately causes high mortality and morbidity. The in-hospital mortality rate for patients with SAH remains very high, approximately $12 \%-20 \%$, despite advancements in care during the last few decades. ${ }^{1,2}$ In survivors, functional outcomes are limited, resulting in $40 \%$ of the patients being unable to return to their prior line of work. ${ }^{3}$ One of the common and often devastating neurological effects of SAH in patients is angiographic (cerebral) vasospasm and delayed cerebral ischemia (DCI). ${ }^{4}$ Diagnostic imaging can detect cerebral vasospasm in as many as 70\% of patients with SAH using CT angiography (CTA) or digital subtraction angiography (DSA). Symptomatic vasospasm, however, is reported to develop in $20 \%-30 \%$ of patients with $\mathrm{SAH}^{4,5}$ and a similar rate is cited for radiographic ischemic changes consistent with DCI. ${ }^{6}$ Cerebral vasospasm and DCI both correlate with functional and cognitive outcome of patients with $\mathrm{SAH}^{7-9}$ as well as with systemic complications during the ICU stay. ${ }^{10}$

Angiographic vasospasm and the development of DCI have a complex and incompletely understood interrelationship. Conventional wisdom suggests that vasospasm of the major proximal intracranial vasculature (macrovascular vasospasm) of the anterior and posterior circulation leads to DCI by reducing cerebral perfusion. Evidence suggests, however, that intracranial vasospasm also occurs in the microvasculature, and in the absence of large arterial (macro) vasospasm, which may be alleviated by dihydropyridine drugs. ${ }^{11}$ Indeed, nimodipine, the only prophylactic treatment with level I evidence to support its use, results in improved patient outcomes, with minimal to no effect on angiographic vasospasm. ${ }^{12}$

Nicardipine, another dihydropyridine calcium antagonist, with similar pharmacological properties to nimodipine, did not improve clinical outcome in a phase III trial when administered intravenously to patients with SAH in randomized controlled trials. Prophylactic intravenous nicardipine, however, did reduce angiographic vasospasm without demonstrating clinical benefit in reducing DCI. ${ }^{13,14}$ One theory to explain the reduction in angiographic vasospasm without improved clinical outcome was that systemically administered nicardipine caused hypotension that was detrimental. One strategy to avoid systemic side effects is intrathecal (IT) delivery via an external ventricular drain (EVD), although there is conflicting data as to the efficacy of this approach. ${ }^{15-22}$

In contrast to a purely preventive approach for allcomers, an alternative strategy is early recognition and intervention. This way, treating cerebral vasospasm occurs only when it is diagnosed. Hence, patients who do not de- velop angiographic vasospasm will not be exposed to the treatment and its potential side effects.

In this report, we summarize the efficacy and safety data of patients with SAH treated therapeutically for cerebral vasospasm. We compared the findings to a known established international registry of SAH trials and single-center experiences, namely the Subarachnoid Hemorrhage International Trialists (SAHIT) database. ${ }^{23}$ A propensity scorebased weighted analysis was utilized. We hypothesized that when compared with a similar SAH patient population, the use of IT nicardipine results in lower rates of radiological DCI and improved long-term functional outcome.

\section{Methods \\ Patient Cohort}

A retrospective clinical data collection of hospital records from patients with SAH admitted to Emory University Hospital and discharged between January 1, 2012, and December 31, 2017, formed the basis of this cohort. The IRB at Emory University approved the data collection and quality assurance analysis and waived the need for patient consent. All patients with a discharge diagnosis of SAH during this time frame were screened. Exclusion criteria included a definite nonaneurysmal etiology (e.g., traumatic SAH, arteriovenous malformation, etc.), while aneurysmal and idiopathic (angio-negative) SAH were included.

Detailed demographic data, clinical assessments, and clinical data, including transcranial Doppler (TCD) and imaging, were obtained from the individual chart review. TCDs were measured daily, 7 days a week. If TCD was not available (for example, because of a lack of bone sonographic windows, or machine availability), surveillance CTA was used at a frequency determined by the clinical team. Standard of care for these patients followed common guidelines, ${ }^{24}$ and included nimodipine, daily statin, maintaining normal magnesium levels, and euvolemia. If hypotension was noted, the usual nimodipine regimen of $60 \mathrm{mg}$ every 4 hours was changed to $30 \mathrm{mg}$ every 2 hours. In rare refractory hypotension, the treatment was further reduced to $30 \mathrm{mg}$ every 4 hours or even paused.

\section{Treatment With Nicardipine}

Treatment with IT nicardipine was initiated after the attending physician (neurocritical care and vascular neurosurgery) determined that radiological/sonographic vasospasm was clinically important. The decision was based on a combination of data that included the neurological examination, TCD velocities and trends, CTA, and baseline risk factors (such as age, smoking status, and modified Fisher grade). No specific cutoff or protocol was used, and therefore some variability occurred in the diagnosis of va- 
sospasm. Clinically relevant cerebral vasospasm prompted interventions that included induced hypertension, cerebral perfusion pressure management, angiography for intraarterial infusion of vasodilators or balloon angioplasty, and IT nicardipine. We defined a subgroup of patients who developed vasospasm (vasospasm compared with no vasospasm, hence no clinically relevant vasospasm determination in real time), as well as a third subgroup of patients who were treated with IT nicardipine (all within the vasospasm group).

The off-label initial IT nicardipine regimen was $4 \mathrm{mg}$ in $2 \mathrm{ml} \mathrm{NaCl} 0.9 \%$ solution administered every 8 hours, and the EVD was flushed with 1-2 $\mathrm{ml}$ of $\mathrm{NaCl} 0.9 \%$ and remained clamped for 30 minutes after nicardipine injection. The EVD was present as part of standard care prior to the decision on IT nicardipine treatment. A decision to change the regimen (increase or decrease dose and/or frequency) was made by the clinical team based on the neurological examination, daily TCD results trend, and CTA, if available.

\section{Radiological Data}

Each brain and vascular imaging was assessed by two study neurologists who were blinded to avoid bias (O. Sadan, D.P., J. Kraft, C.P., S.M., F.A., P.A., and A.T.). Raters were not aware of the vasospasm intervention, severity of vasospasm, or clinical outcome. Radiological DCI was defined as any new ischemic lesion that was not apparent on a scan $24-48$ hours after the aneurysm repair procedure and could not be attributed to another etiology within 6 weeks of the bleeding event. ${ }^{6}$ In the case of any disagreement among the evaluators, the findings were discussed to reach a consensus.

The TCD data were available for analysis from November 2014 onward (when the TCD reports became digitally stored). TCD velocities were acquired daily by a single operator $(n=356)$. The effect of IT nicardipine was measured as the daily change in mean velocities and Lindegaard ratio. ${ }^{25}$ Response index was defined as the ratio between the average TCD velocity in the middle cerebral artery (MCA) territory 24 hours after treatment and the velocity before treatment was initiated. The Supplementary Material reports the CTA information.

\section{Safety}

CSF was routinely obtained three times a week for surveillance chemistry and cultures. Bacterial ventriculitis was defined as the presence of a positive CSF culture that warranted a complete course of antibiotic treatment. Contamination, specifically an immediate sterile repeated culture resulting in stopping antibiotic care, was not defined as ventriculitis.

Outcome measures and complications were derived from the chart review. Long-term functional outcome defined by modified Rankin Scale (mRS) score was obtained from the clinic visit following the acute admission.

\section{Comparative Cohort}

A comparative cohort was generated from the SAHIT database. Anonymized patient-level information was ag- gregated by selecting the specific data sets from the SAHIT database that met the following criterion: patients treated after the International Subarachnoid Aneurysm Trial (ISAT), ${ }^{26}$ to avoid the influence of bias caused by the increased rate of clipping versus endovascular repair of the aneurysm. We also defined a minimum set of parameters available: World Federation of Neurosurgical Societies (WFNS) grade, diagnosis of vasospasm, clinical DCI, radiological DCI (infarct), and long-term functional outcome.

\section{Statistical Analysis}

Descriptive statistics for continuous data are presented as either mean $\pm \mathrm{SD}$ or as median and interquartile range (IQR). Categorical data are presented as counts and percentages. Comparison of continuous outcomes between two groups (e.g., Emory and SAHIT) was made using two-sample t-tests or Wilcoxon rank-sum tests, as appropriate. Comparison of continuous outcomes with more than two groups was performed using ANOVA with post hoc Scheffe testing. Nominal outcomes were compared by a chi-square test for independence and Fisher's exact test. Risk factors associated with binary and ordinal outcomes were assessed with multiple logistic regression or ordinal regression analysis, respectively. Results are given as odds ratios (ORs) with $95 \%$ confidence intervals (CIs). Propensity score-based comparative analysis ${ }^{27,28}$ and the subsequent sensitivity analysis are detailed in the Supplementary Material (Supplementary Fig. 2, Supplementary Tables 4 and 5).

\section{Results \\ Patient Cohort}

A total of 1351 patients were discharged from Emory University Hospital neurosciences ICU with nontraumatic SAH between 2012 and 2017. Of these, 993 (73.5\%) were aneurysmal and 358 (26.5\%) were idiopathic. Demographic information regarding the cohort is detailed in Table 1. In this cohort, $36.4 \%(n=492)$ were diagnosed with clinically relevant cerebral vasospasm by means of clinical change and blood flow velocity increase on TCD, or by angiographic evidence on CTA or DSA reports. Of the patients diagnosed with vasospasm, $85.8 \%(n=422)$ were treated with IT nicardipine (Table 1). In the group that was diagnosed with vasospasm and not treated with IT nicardipine, $67.1 \%(n=47)$ did not have an EVD to allow its administration.

Prior to and in parallel with IT nicardipine treatment, patients were treated with cerebral perfusion pressure management as per the standard of care. In the group treated with IT nicardipine, 59\% required a vasopressor infusion to maintain the pressure at target. Furthermore, $21 \%$ were sent for a DSA as a rescue treatment, yet only $3 \%$ needed a second treatment.

\section{Administration of IT Nicardipine}

Patients treated with IT nicardipine were delineated from patients who did not receive the treatment by the known risk factors for cerebral vasospasm (sex, age, smoking status, higher-grade hemorrhage, and aneurysmal etiology; Table 1). Treatment with IT nicardipine began on 
TABLE 1. Patient and clinical characteristics of the Emory cohort

\begin{tabular}{|c|c|c|c|c|c|}
\hline Variable & All & $\begin{array}{c}\text { No Vasospasm, No IT } \\
\text { Nicardipine }\end{array}$ & $\begin{array}{c}\text { Vasospasm, No IT } \\
\text { Nicardipine }\end{array}$ & $\begin{array}{l}\text { Vasospasm + IT } \\
\text { Nicardipine }\end{array}$ & p Value* \\
\hline No. of patients & $1351(100.00)$ & $859(63.6)$ & $70(5.2)$ & $422(31.2)$ & \\
\hline Mean age $\pm S D$, yrs & $54.5 \pm 13.8$ & $56.7 \pm 14.1$ & $47.5 \pm 11.7$ & $51.1 \pm 12.4$ & $<0.001$ \\
\hline Females & $906(67.1)$ & $548(63.8)$ & $50(71.4)$ & $308(73.0)$ & 0.001 \\
\hline Race & & & & & 0.518 \\
\hline African American & $452(33.5)$ & $275(32.0)$ & $26(37.1)$ & $151(35.8)$ & \\
\hline White & $581(43.0)$ & $383(44.6)$ & $31(44.3)$ & $167(39.6)$ & \\
\hline Asian & $34(2.5)$ & $20(2.3)$ & $2(2.9)$ & $12(2.8)$ & \\
\hline Hispanic/Latino & $39(2.9)$ & $23(2.7)$ & $4(5.7)$ & $12(2.8)$ & \\
\hline Other/unknown & $245(18.1)$ & $158(18.4)$ & $7(10.0)$ & $80(19.0)$ & \\
\hline Diabetes & $167(12.4)$ & $129(15.0)$ & $7(10.0)$ & $31(7.3)$ & $<0.001$ \\
\hline Smoker & $378(28.0)$ & $222(25.8)$ & $17(24.3)$ & $139(32.9)$ & 0.008 \\
\hline Hypertension & $759(56.2)$ & $505(58.8)$ & 19 (27.1) & $235(55.7)$ & 0.291 \\
\hline Coronary artery disease & $93(6.9)$ & $70(8.1)$ & $1(1.4)$ & $22(5.2)$ & 0.056 \\
\hline Hyperlipidemia & $216(16.0)$ & $167(19.4)$ & $6(8.6)$ & $43(10.2)$ & $<0.001$ \\
\hline Surgical treatment & & & & & $<0.001$ \\
\hline Clip & $241(17.8)$ & $97(11.3)$ & $16(22.9)$ & $128(30.3)$ & \\
\hline Endovascular & $622(46.0)$ & $346(40.3)$ & $18(25.7)$ & $258(61.1)$ & \\
\hline Idiopathic (angio-negative) & $358(26.5)$ & $297(34.6)$ & $34(48.6)$ & $27(6.4)$ & \\
\hline No treatment & $130(9.6)$ & $119(13.8)$ & $2(2.9)$ & $9(2.1)$ & \\
\hline WFNS grade & & & & & $<0.001$ \\
\hline I & $604(44.7)$ & $451(52.5)$ & $49(70.0)$ & $104(24.6)$ & \\
\hline II-III & $343(25.4)$ & $187(21.8)$ & $11(15.7)$ & $145(34.4)$ & \\
\hline IV-V & $404(29.9)$ & $221(25.7)$ & $10(14.3)$ & $173(41.0)$ & \\
\hline Modified Fisher grade & & & & & $<0.001$ \\
\hline 0 & $26(2.0)$ & $24(2.9)$ & $1(1.4)$ & $1(0.2)$ & \\
\hline 1 & $161(12.3)$ & $131(16.0)$ & $19(27.1)$ & $11(2.6)$ & \\
\hline 2 & $117(8.9)$ & $83(10.2)$ & $5(7.1)$ & $29(6.9)$ & \\
\hline 3 & $231(17.7)$ & $152(18.6)$ & $22(31.4)$ & $57(13.5)$ & \\
\hline 4 & $772(59.1)$ & $426(52.2)$ & $23(32.9)$ & $323(76.5)$ & \\
\hline
\end{tabular}

Values are presented as the number (\%) unless otherwise indicated.

* No vasospasm versus vasospasm treated with IT nicardipine.

mean postadmission day $5.8 \pm 2.8$ and lasted for an average of $8.3 \pm 5.1$ days (Fig. 1). The median number of doses of IT nicardipine was 20 (IQR 18). The initial dose was 4 $\mathrm{mg}$ every 8 hours in $75.4 \%$ of the cases (range of $2 \mathrm{mg} \mathrm{ev}$ ery 12 hours to $4 \mathrm{mg}$ every 4 hours). In $79.6 \%$ of the cases, the initial regimen chosen was not increased. In cases of suboptimal response or temporary response, defined by either clinical examination or radiological worsening on TCD or CTA, the dose was increased in $1.5 \%$ (e.g., from 2 to $4 \mathrm{mg}$ ), and the frequency increased in $19.5 \%$ of the cases (e.g., from every 8 to every 6 hours). This change, if it occurred, happened on average on day $2.6 \pm 2.3$ of treatment. Out of concern for abrupt discontinuation promoting a rebound effect, the local practice pattern was to wean off of treatment in $75.1 \%$ of cases over 2-4 days.

\section{Change in Proximal Artery Vasospasm}

TCD data was available for 198 (46.9\%) of 422 patients who received treatment with IT nicardipine, and 338 pa- tients (39.3\%) who did not develop clinically significant vasospasm (Fig. 2A). On the day of IT nicardipine treatment initiation, the maximal mean MCA velocity was over 100 $\mathrm{cm} / \mathrm{sec}$ in $79.5 \%$ of the patients (95\% CI $73.2 \%-85.8 \%$ ), above $90 \mathrm{~cm} / \mathrm{sec}$ in the anterior cerebral artery (ACA) in $74.4 \%$ (95\% CI $67.5 \%-81.2 \%$ ), and above $70 \mathrm{~cm} / \mathrm{sec}$ in the basilar artery (BA) in $47.7 \%(95 \%$ CI $39.8 \%-55.6 \%)$. When available, CTA-based diagnosis of vasospasm was also analyzed, which demonstrated moderate to severe vasospasm in $82.8 \%$ of the treated patients (see Supplementary Methods and Supplementary Fig. 1).

Treatment was associated with a reduction of mean velocities in all main arteries (ACA, MCA, BA) after 1 day of treatment (relative reduction of $12.9 \%$ on average in the MCA, $13.0 \%$ in the ACA, and $19.3 \%$ in the basilar territory, $\mathrm{p}<0.01$; Fig. 2B). This effect remained through the treatment, until the velocities normalized and plateaued. When analyzing the response in treated patients, more than $77.3 \%$ of patients showed at least 1 of the 3 main ar- 

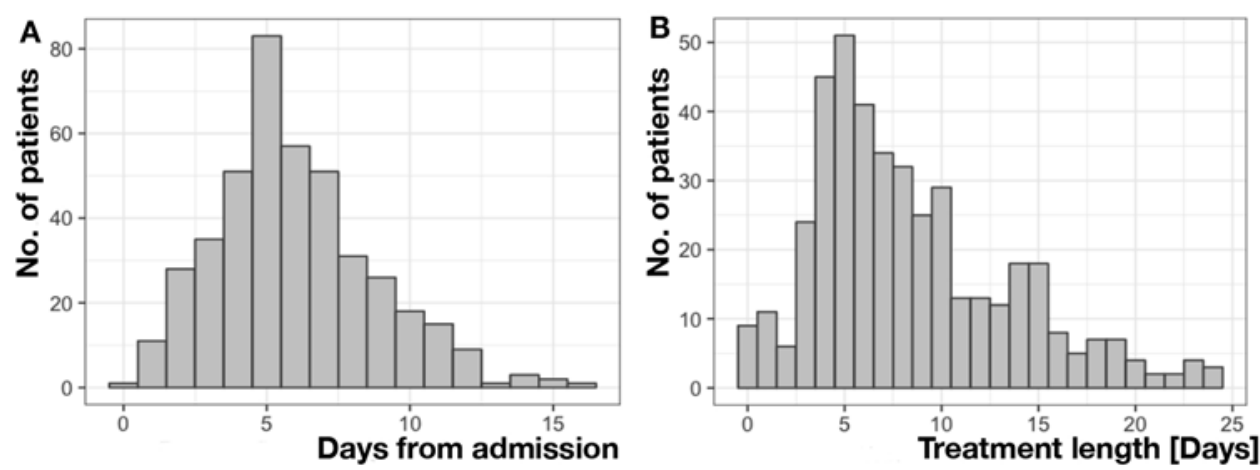

FIG. 1. Administration of IT nicardipine. A: Histogram showing the frequency of treatment initiation with IT nicardipine by admission day. B: Histogram showing the length of treatment in days.

teries experienced TCD velocity decrease within 24 hours of treatment (Fig. 2C).

Increased TCD velocities were correlated to the risk of DCI (Fig. 3). A positive response to IT nicardipine by means of decreasing blood velocity rates, however, did not correlate with either decreased incidence of DCI or improvement in long-term functional status (Fig. 4).

\section{Safety}

Bacterial ventriculitis was diagnosed in $2.9 \%$ of the patients who required an EVD in our cohort $(n=866)$. The prevalence of ventriculitis rate in patients who were treated with IT nicardipine was similar to those who did not (3.1\% vs $2.7 \%$, respectively; OR $1.15,95 \%$ CI $0.52-2.54$ ). A ventriculoperitoneal shunt (VPS) was required because of persistent hydrocephalus in $14.2 \%$ of patients who had an EVD placed. Significantly more patients treated with IT nicardipine required a VPS compared with those who were not treated with IT nicardipine $(19.9 \%$ vs $8.8 \%$, p < 0.01 ). In a multiple logistic regression analysis, adjusting for demographics and risk factors, IT nicardipine administration was associated with an increased need for a VPS (OR 2.54, 95\% CI 1.68-3.83).

\section{Comparative Outcome Analysis}

The outcome analysis of the Emory cohort is detailed in Supplementary Table 1. Due to the retrospective nature of this study, a relevant control group within the cohort was not available. Therefore, we next performed a comparative analysis with patients from the SAHIT database. As detailed in the Supplementary Methods and Supplementary Results, following propensity-score average treatment effect for treated-based weighting, we analyzed data from 446 patients of the SAHIT data set who were similar to the 422 patients from the Emory cohort who were treated with IT nicardipine (Supplementary Tables 2 and 3). The group treated with IT nicardipine had a lower rate of DCI, higher proportion of patients with favorable long-term functional outcome, and lower rates of bacterial ventriculitis (Table 2). The proportion of patients requiring a VPS in the weighted SAHIT group was $28.4 \%$, which was higher compared to the IT nicardipine group. Because information regarding a VPS was available for only $25.1 \%$ of the SAHIT cohort, we did not include it in the logistic regression analysis.
Because patients with idiopathic SAH tend to have a less morbid clinical course, we performed a separate sensitivity analysis in which these patients were excluded from both the IT nicardipine $(\mathrm{n}=27)$ and SAHIT $(\mathrm{n}=$ 101) cohorts (Supplementary Table 5). Excluding angionegative SAH did not modify the odds of favorable outcomes between cohorts.

\section{Discussion}

This study is a retrospective description of the largest cohort to date involving the use of IT nicardipine in patients with nontraumatic SAH to treat cerebral vasospasm and prevent DCI. The results corroborate known risk factors for cerebral vasospasm and DCI, including younger age, female sex, tobacco abuse, and higher WFNS and modified Fisher grades. Following comparison with the SAHIT database, the data indicate that for patients diagnosed with clinically relevant vasospasm, treatment with IT nicardipine reduced proximal artery vasospasm, and was associated with a lower rate of DCI and with a higher rate of good functional outcome. Moreover, IT nicardipine was not associated with higher infection rates, although it was associated with higher rate of VPS insertion.

Historically, vasospasm was noted on diagnostic cerebral angiography following SAH in proximal arteries. ${ }^{29}$ The pathophysiology of vasospasm continues to be incompletely understood; is complex; and includes vasoconstriction, in part related to hemoglobin from hemolyzed red blood cells,$^{30}$ nitric oxide, and endothelin pathways. ${ }^{31}$ For the bedside clinician, identifying and intervening upon cerebral vasospasm is challenging. Radiological evidence for vasospasm is common in patients with SAH. A correlation exists between vasospasm and later development of DCI. Multiple interventions in the literature, however, demonstrated improvement of vasospasm without preventing DCI, or affecting functional outcome. ${ }^{16,32-34}$ In hindsight, several commonalities emerge from reviewing prior negative studies. In most studies, the treatment was prophylactic, and perhaps one dose does not fit all. ${ }^{13,35} \mathrm{An}$ other issue is targeting resolution of vasospasm in earlier phase studies, and later failing in phase III studies when patient outcomes are the clinical end point. That was most notably relevant for the recent NEWTON-2 (Nimodipine Microparticles to Enhance Recovery While Reducing 

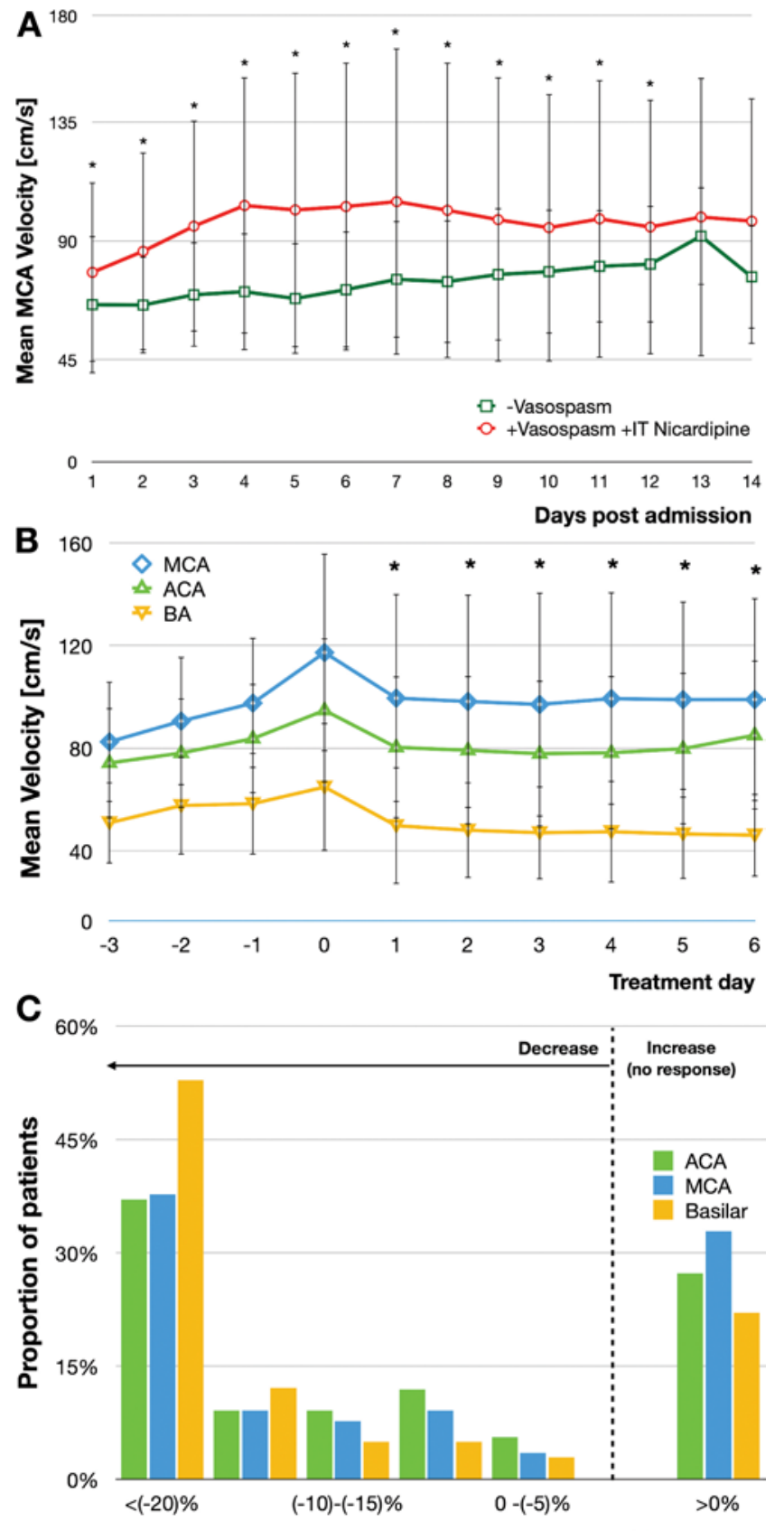

A $50 \%$

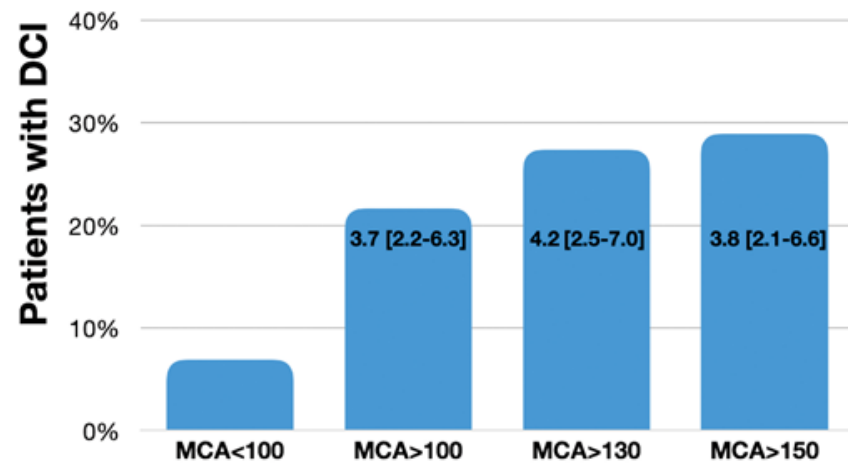

B 50\%
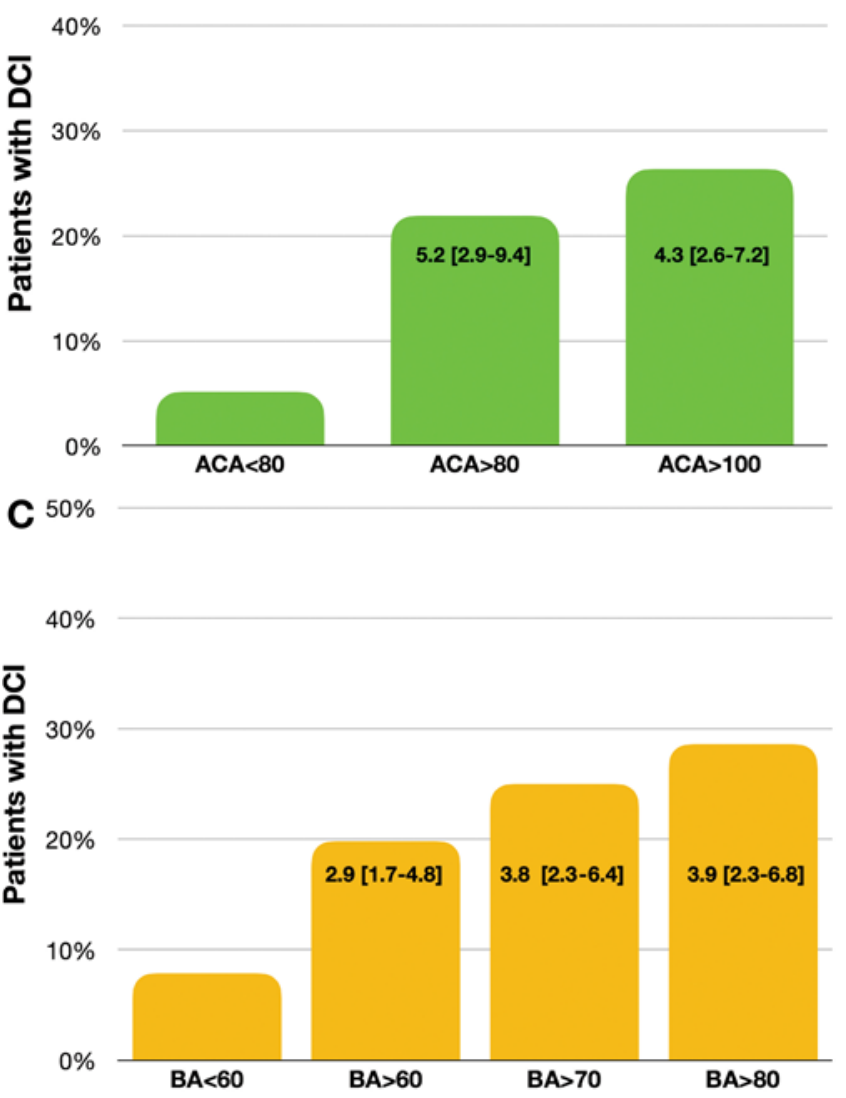

FIG. 2. Change in blood flow velocities measured by TCD in response to IT nicardipine. TCD was not a single criterion to select patients for treatment, and no single threshold was used. Patient selection for treatment was based on risk factors, change on examination, TCD, and other radiological data, as detailed in the Methods section. For each artery (MCA, ACA, BA), the average measurements of the mean velocity at the different depths were calculated. Results are presented as mean \pm SD. A: The timeline of cerebral vasospasm: comparison of the average mean MCA velocities between the group diagnosed with vasospasm and treated with IT nicardipine, and the group that did not have clinically relevant vasospasm ( ${ }^{*} p<0.05$ between groups). B: Change in average mean velocity in comparison to the initiation day of IT nicardipine by artery (day 0; * $p<0.05$ compared to day 0). C: Distribution of the magnitude of response in blood flow velocities within the first day (24 hours) of treatment (change in mean velocities between day 0 and day 1 , per artery, in percentage) in patients treated with IT nicardipine.

FIG. 3. Correlation between TCD velocity and outcome. Proportion of $\mathrm{DCl}$ according to the maximal MCA velocity $(A, n=162)$, maximal $A C A$ velocity $(B, n=162)$, and maximal $B A$ velocity $(C, n=158)$. ORs [95\% $\mathrm{Cls}$ ] based on unadjusted binary regression analysis are presented in each bar.

Toxicity After Subarachnoid Hemorrhage) study. In the NEWTON-2 trial, a single dose of slow-release nimodipine was administered through an EVD. The study was stopped prematurely when a planned interim analysis suggested the study had a low likelihood of meeting its primary outcome. However, it did demonstrate a reduced rate of proximal vessel vasospasm, but not improvement 

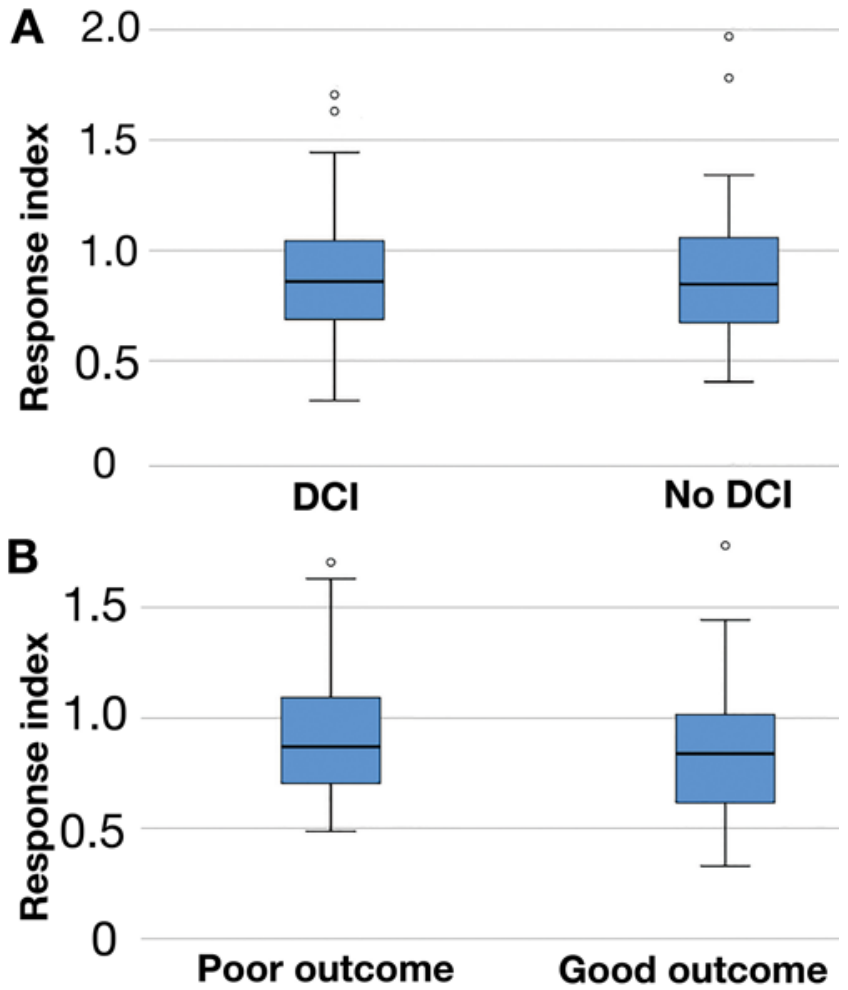

FIG. 4. TCD MCA response index (mean MCA velocity on day 1 of treatment/day 0 ) and correlation to clinical outcomes. A decrease in mean velocity in response to treatment is noted as index $<1$. A: Response index was compared in patients with and without $\mathrm{DCl}$ and demonstrated no statistically significant difference $(n=162)$. B: Response index showed no statistically significant difference in comparing patients with good long-term functional outcome (mRS score $\leq 2$ ) with the poor outcome group (mRS score $>2, n=123$ ). Blue boxes represent quartiles $1-3$. Open circles are outliers.

in outcome. A trend toward improvement was noted in the more severely ill patients, suggesting that at least in part, this failure was related to patient selection. ${ }^{16}$ To summarize, to date, there is no proven treatment available that results in reduced vasospasm, reduced DCI, and improved patient outcome in patients with cerebral vasospasm from nontraumatic SAH..$^{12,36}$

Our experience builds on these lessons, with a treatment regimen focused on reactive, not prophylactic, treatment driven by patient response to a given treatment regimen, which is similar to prior smaller series reported.$^{37}$ IT nicardipine in this report was largely triggered by radiological data (TCD, CTA, or DSA). Given the correlation of radiological vasospasm with DCI (Fig. 3), this approach likely improved patient selection for those at high risk.

IT nicardipine was administered in addition to standard-of-care prophylactic treatment (oral nimodipine) and commonly used interventions such as cerebral perfusion pressure management. However, only a minority of the patients required invasive interventions such as intraarterial infusion or angioplasty. This result corresponds with our recent report showing a reduction in the rate of repeated DSA in the past 15 years. ${ }^{2}$ The dose and length of treatment were titrated to effect, which was determined by improved neurological examination and/or improved radiological vasospasm. This approach, however, introduces issues arising from the spectrum of clinical decision-making in a retrospective cohort without a formal protocol. As such, the definition of clinically relevant vasospasm varied among clinicians. The majority of patients demonstrated radiological signs of mild to moderate vasospasm in more than one vessel when IT nicardipine treatment was initiated. In some cases, however, initiation began with less severe spasm than others and in different distributions (one focal severe stenosis versus milder yet widespread vasospasm).

Cerebral vasospasm is thought to occur on both the macro- and microvascular levels. Most retrospective results so far, ${ }^{38-40}$ including several systemic reviews, ${ }^{37,41,42}$ have suggested a beneficial effect of IT nicardipine on macrovascular blood velocities, similar to our report. However, evidence has accumulated that loss of microvasculature autoregulation and consequent decrease in regional blood flow are also related to DCI. ${ }^{43}$ Whether IT nicardipine has an effect on the microcirculation, similar to the one it has on the proximal vessel level, is unclear. One attempt to measure the microcirculatory effect measured the effect of IT nicardipine on tissue oxygenation $\left(\mathrm{PbO}_{2}\right)$ and metabolites (by microdialysis), which did not demonstrate a robust effect. ${ }^{44}$ The effort was limited, however, by the local measurement and probe location. The observed clinical benefit in the current report (Table 2) did not correlate with improvement in macrovascular vasospasm (Figs. 1 and 3), suggesting that IT nicardipine may improve functional outcomes by modifying microvascular vasospasm.

We also attempted to identify complications from frequently accessing an EVD. The overall rate of bacterial ventriculitis was quite low in our cohort compared to the SAHIT cohort, although no routine IT intervention occurred in the SAHIT cohort. Even when examining the rate of infection within our cohort, and comparing those who were treated with IT nicardipine versus those who were not, the IT treatment did not contribute to an increased

TABLE 2. Clinical outcomes comparison between the IT nicardipine-treated group and the weighted group from the SAHIT data set

\begin{tabular}{lccc}
\hline \multicolumn{1}{c}{ Outcome } & IT Nicardipine & SAHIT (post-weighting) & OR (95\% Cl) \\
\hline Favorable outcome at 3 mos & $62.5 \%$ & $43.6 \%$ & $2.17(1.61-2.91)$ \\
\hline DCl & $20.2 \%$ & $28.7 \%$ & $0.61(0.44-0.84)$ \\
\hline Bacterial ventriculitis & $3.1 \%$ & $10.1 \%$ & $0.29(0.15-0.54)$ \\
\hline${ }^{*}$ For IT nicardipine treatment & & &
\end{tabular}


rate of infection. Prior reports regarding IT nicardipine treatment described an infection rate of approximately $6 \%$, nearly double those reported in this publication. ${ }^{42}$ Whether the relatively low rate of infection is reproducible, and what the true added risk of bacterial ventriculitis is with the addition of IT nicardipine treatment, remain unclear. The rate of VPS placement in our cohort was also lower compared with the SAHIT cohort, although too many data points were missing to compare the two comprehensively. In our cohort, a clear association was observed between IT nicardipine and the further need for a VPS, even after controlling for the relevant risk factors. At this time, it is unclear whether this effect is a result of an IT intervention per se, a reaction to nicardipine or its formulation, or other explanations. Future studies that compare nicardipine with a placebo may provide insight on this question.

\section{Limitations of the Study}

This study has limitations arising from its retrospective nature and lack of having a formal protocol. When compared with our own group's prior description of this intervention, over the course of several years, the threshold for initiation seems lower, ${ }^{38}$ and the overall definition of clinically significant vasospasm varied. In lieu of an internal control group, we used an external database. The SAHIT data set involves diverse cohorts, which include prior clinical trial data and single-center cohorts. To reduce possible bias, we did not take into account patient cohorts that preceded the ISAT, ${ }^{26}$ because following that study the rate of endovascular treatment for securing aneurysms became more abundant and potentially reduced rates of DCI. On the one hand, this database is based on practice without a formal protocol, and therefore could have biases. On the other hand, this diversity could mean that the patient data are closer to real-life experience, similar to our cohort.

Thus far, prior reports and systematic reviews $\mathrm{s}^{37,41,42}$ regarding IT nicardipine have lacked long-term outcomes. Our report suggests that a beneficial long-term effect may exist. Despite the significant steps taken to avoid bias such as using propensity score-based comparison, this analysis cannot replace a prospective controlled trial.

\section{Conclusions}

The current study describes the largest SAH patient cohort to date that received IT nicardipine treatment to alleviate vasospasm and reduce DCI. These data show that this treatment was associated with a reduced rate of DCI and increased proportion of patients with long-term good functional outcome. The treatment was also associated with an increased rate of VPS placement. The results are limited by their retrospective nature. Because of the paucity of effective and proven treatments for cerebral vasospasm and prevention of DCI, these results hold promise that will need to be verified in a prospective, randomized controlled clinical trial to test the efficacy of IT nicardipine treatment for post-SAH cerebral vasospasm.

\section{Acknowledgments}

We would like to thank the SAHIT collaborators (Jeroen Boogaarts [Radboud University Medical Center], Walter van den Bergh [University Medical Center Utrecht], Hitoshi Fukuda [Kurashiki Central Hospital], Daniel Hanggi [Heinrich Heine University], Peter Le Roux [University of Pennsylvania], Benjamin Lo [University of Toronto], Stephan Mayer [Columbia University], Andrew Molyneux [Oxford University], Audrey Quinn [The General Infirmary, Leeds], Gabriel Rinkel [University Medical Center Utrecht], Tom Schweizer [University of Toronto], Jose Suarez [Johns Hopkins University], Michael Todd [University of Iowa], Mervyn Vergouwen [University Medical Center Utrecht], and George Wong [Chinese University of Hong Kong]), who created the comparative database used as the comparative group in this study.

\section{References}

1. Udy AA, Vladic C, Saxby ER, et al. Subarachnoid hemorrhage patients admitted to intensive care in Australia and New Zealand: a multicenter cohort analysis of in-hospital mortality over 15 years. Crit Care Med. 2017;45(2):e138e145.

2. Samuels OB, Sadan O, Feng C, et al. Aneurysmal subarachnoid hemorrhage: trends, outcomes, and predictions from a 15-year perspective of a single neurocritical care unit. Neurosurgery. 2021;88(3):574-583.

3. Al-Khindi T, Macdonald RL, Schweizer TA. Cognitive and functional outcome after aneurysmal subarachnoid hemorrhage. Stroke. 2010;41(8):e519-e536.

4. Vergouwen MDI. Vasospasm versus delayed cerebral ischemia as an outcome event in clinical trials and observational studies. Neurocrit Care. 2011;15(2):308-311.

5. Wartenberg KE, Schmidt JM, Claassen J, et al. Impact of medical complications on outcome after subarachnoid hemorrhage. Crit Care Med. 2006;34(3):617-624.

6. Vergouwen MDI, Vermeulen M, van Gijn J, et al. Definition of delayed cerebral ischemia after aneurysmal subarachnoid hemorrhage as an outcome event in clinical trials and observational studies: proposal of a multidisciplinary research group. Stroke. 2010;41(10):2391-2395.

7. Springer MV, Schmidt JM, Wartenberg KE, et al. Predictors of global cognitive impairment 1 year after subarachnoid hemorrhage. Neurosurgery. 2009;65(6):1043-1051.

8. Macdonald RL, Hunsche E, Schüler R, et al. Quality of life and healthcare resource use associated with angiographic vasospasm after aneurysmal subarachnoid hemorrhage. Stroke. 2012;43(4):1082-1088.

9. Pegoli M, Mandrekar J, Rabinstein AA, Lanzino G. Predictors of excellent functional outcome in aneurysmal subarachnoid hemorrhage. J Neurosurg. 2015;122(2):414-418.

10. Frontera JA, Fernandez A, Schmidt JM, et al. Defining vasospasm after subarachnoid hemorrhage: what is the most clinically relevant definition? Stroke. 2009;40(6):1963-1968.

11. Foreman B. The pathophysiology of delayed cerebral ischemia. J Clin Neurophysiol. 2016;33(3):174-182.

12. Connolly ES Jr, Rabinstein AA, Carhuapoma JR, et al. Guidelines for the management of aneurysmal subarachnoid hemorrhage: a guideline for healthcare professionals from the American Heart Association/American Stroke Association. Stroke. 2012;43(6):1711-1737.

13. Haley EC Jr, Kassell NF, Torner JC. A randomized trial of nicardipine in subarachnoid hemorrhage: angiographic and transcranial Doppler ultrasound results. A report of the Cooperative Aneurysm Study. J Neurosurg. 1993;78(4):548-553.

14. Flamm ES, Adams HP Jr, Beck DW, et al. Dose-escalation study of intravenous nicardipine in patients with aneurysmal subarachnoid hemorrhage. J Neurosurg. 1988;68(3):393-400.

15. Hänggi D, Etminan N, Aldrich F, et al. Randomized, open-label, phase $1 / 2$ a study to determine the maximum tolerated dose of intraventricular sustained release nimodipine for subarachnoid hemorrhage (NEWTON [Nimodipine Micropar- 
ticles to Enhance Recovery While Reducing Toxicity After Subarachnoid Hemorrhage]). Stroke. 2017;48(1):145-151.

16. Carlson AP, Hänggi D, Wong GK, et al. Single-dose intraventricular nimodipine microparticles versus oral nimodipine for aneurysmal subarachnoid hemorrhage. Stroke. 2020;51(4): 1142-1149.

17. Barth M, Capelle H-H, Weidauer S, et al. Effect of nicardipine prolonged-release implants on cerebral vasospasm and clinical outcome after severe aneurysmal subarachnoid hemorrhage: a prospective, randomized, double-blind phase IIa study. Stroke. 2007;38(2):330-336.

18. Kasuya H. Clinical trial of nicardipine prolonged-release implants for preventing cerebral vasospasm: multicenter cooperative study in Tokyo. Acta Neurochir Suppl (Wien). 2011; 110(Pt 2):165-167.

19. Kasuya H, Onda H, Sasahara A, et al. Application of nicardipine prolonged-release implants: analysis of 97 consecutive patients with acute subarachnoid hemorrhage. Neurosurgery. 2005;56(5):895-902.

20. Kasuya H, Onda H, Takeshita M, et al. Efficacy and safety of nicardipine prolonged-release implants for preventing vasospasm in humans. Stroke. 2002;33(4):1011-1015.

21. Kuroi Y, Ohbuchi H, Arai N, et al. Twelve-year single critical care center experience of nicardipine prolonged-release implants in patients with subarachnoid hemorrhage: a propensity score matching analysis. J Neurointerv Surg. 2020;12(8): 774-776.

22. Barth M, Pena P, Seiz M, et al. Feasibility of intraventricular nicardipine prolonged release implants in patients following aneurysmal subarachnoid haemorrhage. Br J Neurosurg. 2011;25(6):677-683.

23. Jaja BNR, Saposnik G, Lingsma HF, et al. Development and validation of outcome prediction models for aneurysmal subarachnoid haemorrhage: the SAHIT multinational cohort study. BMJ. 2018;360:j5745.

24. Bederson JB, Connolly ES Jr, Batjer HH, et al. Guidelines for the management of aneurysmal subarachnoid hemorrhage: a statement for healthcare professionals from a special writing group of the Stroke Council, American Heart Association. Stroke. 2009;40(3):994-1025.

25. Lindegaard KF, Nornes H, Bakke SJ, et al. Cerebral vasospasm after subarachnoid haemorrhage investigated by means of transcranial Doppler ultrasound. Acta Neurochir Suppl (Wien). 1988;42:81-84.

26. Molyneux A, Kerr R, Stratton I, et al. International Subarachnoid Aneurysm Trial (ISAT) of neurosurgical clipping versus endovascular coiling in 2143 patients with ruptured intracranial aneurysms: a randomised trial. Lancet. 2002;360(9342): 1267-1274.

27. Brookhart MA, Schneeweiss S, Rothman KJ, et al. Variable selection for propensity score models. Am J Epidemiol. 2006; 163(12):1149-1156.

28. Rubin DB. Using propensity scores to help design observational studies: application to the tobacco litigation. Health Serv Outcomes Res Methodol. 2001;2(3):169-188.

29. Steiner L, Forster DM, Bergvall U, Carlson LA. Effect of prostaglandin E 1 on cerebral circulatory disturbances following subarachnoid haemorrage in man. Neuroradiology. 1972;4(1):20-24.

30. Toda N, Shimizu K, Ohta T. Mechanism of cerebral arterial contraction induced by blood constituents. J Neurosurg. 1980;53(3):312-322.

31. Ciurea AV, Palade C, Voinescu D, Nica DA. Subarachnoid hemorrhage and cerebral vasospasm-literature review. $J$ Med Life. 2013;6(2):120-125.

32. Etminan N, Vergouwen MDI, Ilodigwe D, Macdonald RL. Effect of pharmaceutical treatment on vasospasm, delayed cerebral ischemia, and clinical outcome in patients with aneurysmal subarachnoid hemorrhage: a systematic review and meta-analysis. J Cereb Blood Flow Metab. 2011;31(6): 1443-1451.

33. Dorhout Mees SM, Algra A, Vandertop WP, et al. Magnesium for aneurysmal subarachnoid haemorrhage (MASH-2): a randomised placebo-controlled trial. Lancet. 2012;380(9836): 44-49.

34. Macdonald RL, Higashida RT, Keller E, et al. Randomized trial of clazosentan in patients with aneurysmal subarachnoid hemorrhage undergoing endovascular coiling. Stroke. 2012; 43(6):1463-1469.

35. Koyanagi M, Fukuda H, Lo B, et al. Effect of intrathecal milrinone injection via lumbar catheter on delayed cerebral ischemia after aneurysmal subarachnoid hemorrhage. $\mathrm{J} \mathrm{Neu}$ rosurg. 2018;128(3):717-722.

36. Weyer GW, Nolan CP, Macdonald RL. Evidence-based cerebral vasospasm management. Neurosurg Focus. 2006;21(3):E8.

37. Dodson V, Majmundar N, El-Ghanem M, et al. Intracranial administration of nicardipine after aneurysmal subarachnoid hemorrhage: a review of the literature. World Neurosurg. 2019;125:511-518.e1.

38. Webb A, Kolenda J, Martin K, et al. The effect of intraventricular administration of nicardipine on mean cerebral blood flow velocity measured by transcranial Doppler in the treatment of vasospasm following aneurysmal subarachnoid hemorrhage. Neurocrit Care. 2010;12(2):159-164.

39. Lu N, Jackson D, Luke S, et al. Intraventricular nicardipine for aneurysmal subarachnoid hemorrhage related vasospasm: assessment of 90 days outcome. Neurocrit Care. 2012;16(3): $368-375$

40. Suzuki M, Doi M, Otawara Y, et al. Intrathecal administration of nicardipine hydrochloride to prevent vasospasm in patients with subarachnoid hemorrhage. Neurosurg Rev. 2001;24(4):180-184.

41. Stuart D, Christian R, Uschmann H, Palokas M. Effectiveness of intrathecal nicardipine on cerebral vasospasm in nontraumatic subarachnoid hemorrhage: a systematic review. JBI Database Syst Rev Implement Reports. 2018;16(10): 2013-2026.

42. Hafeez S, Grandhi R. Systematic review of intrathecal nicardipine for the treatment of cerebral vasospasm in aneurysmal subarachnoid hemorrhage. Neurocrit Care. 2019;31(2): 399-405.

43. Ohkuma H, Manabe H, Tanaka M, Suzuki S. Impact of cerebral microcirculatory changes on cerebral blood flow during cerebral vasospasm after aneurysmal subarachnoid hemorrhage. Stroke. 2000;31(7):1621-1627.

44. Ko SB, Choi HA, Helbok R, et al. Acute effects of intraventricular nicardipine on cerebral hemodynamics: a preliminary finding. Clin Neurol Neurosurg. 2016;144:48-52.

\section{Disclosures}

The authors report no conflict of interest concerning the materials or methods used in this study or the findings specified in this paper.

\section{Author Contributions}

Conception and design: Sadan, Asbury, Jaja, Macdonald, Cawley, Barrow, Samuels. Acquisition of data: Sadan, Feng, Pearce,

Kraft, Pimentel, Mathew, Akbik, Ameli, Taylor, Danyluk, Martin, Garner, Kolenda, Pujari. Analysis and interpretation of data: Sadan, Waddel, Moore, Feng, Mei, Pearce, Kraft, Pimentel, Mathew, Akbik, Ameli, Taylor, Danyluk, Martin, Garner, Kolenda, Pujari, Jaja, Macdonald, Samuels. Drafting the article: Sadan, Waddel, Moore, Feng, Samuels. Critically revising the article: Moore, Mei, Pearce, Kraft, Pimentel, Akbik, Ameli, Kolenda, Asbury, Jaja, Macdonald, Cawley, Barrow, Samuels. Reviewed submitted version of manuscript: all authors. Approved the final version of the manuscript on behalf of all authors: Sadan. 
Statistical analysis: Waddel, Moore, Feng, Mei. Administrative/ technical/material support: Pujari. Study supervision: Asbury, Macdonald, Cawley, Barrow, Samuels.

\section{Supplemental Information}

Online-Only Content

Supplemental material is available with the online version of the article.

Supplementary Material. https://thejns.org/doi/suppl/10.3171/ 2020.12.JNS203673.

\section{Previous Presentations}

Parts of these results were presented as abstracts at the following meetings: Neurocritical Care Annual Meeting, Vancouver, Canada, September 2019; American Heart Association International Stroke Conference, Los Angeles, California, February 19-21,
2020; and the Society for Critical Care Medicine Annual Meeting, Orlando, Florida, February 16-19, 2020.

\section{Preprint Server}

An earlier version of this article can be found on a preprint server. Preprint server name: medRxiv.

Preprint DOI: 10.1101/2020.08.31.20185181v1.

\section{Correspondence}

Ofer Sadan: Emory University School of Medicine, Atlanta, GA. ofer.sadan@gmail.com. 